\title{
A NOVEL MULTI-ROBOT COORDINATION METHOD USING CAPABILITY CATEGORY
}

\author{
Liu Lin and Zhiqiang Zheng
}

\author{
Department of Automatic Control of Mechatronics and \\ Automation School \\ National University of Defense Technology \\ ChangSha Hunan, 410073, P. R. China \\ Email: liu_double@263.net
}

\begin{abstract}
This paper presents a set of formal descriptions to the multi-robot task allocation problem mainly focusing on the capability category aspect. Both robot and task capability vectors are defined. Based upon the these, both single and multi-robot cooperative task accomplishment conditions are discussed. Based upon the capability category and task accomplishment condition, a novel combinatorial bids method followed with some discussions is proposed. Copyright (c)2005 IFAC
\end{abstract}

Keywords: Multi Robot, Coordination, Capability Category, Combinatorial Bids, Market Based

\section{INTRODUCTION}

Over the past decade, more and more researchers began to shift their interests to the field of multi-robot systems (Arai et al., 2002) (Cao et al., 1997). As researchers design, build, and use cooperative multi-robot systems, they invariably should give answer to a question that which robot should execute which task. This is in fact a multi-robot task allocation problem (MRTA) (Parker, 1998) (Brian and Mataric, 2004).

Lynee E. Parker (Parker, 1998) designed a wellknown ALLIANCE architecture, which is a fully distributed, behavior-based architecture that incorporates the use of mathematically-modeled motivations within each robot to achieve action selection.

Brian P. Gerkey et al. (Brian and Mataric, 2002) presented a dynamic task allocation method for groups of failure-prone autonomous robots. They implemented and tested an auction-based task allocation system: MURDOCH, which was built upon a principled, resource centric, publish/subscribe communication model.

In the distributed artificial intelligence and multiagent domain, many researchers have studied how to coordinate agents with free market based mechanism and much work was done in this domain (Wellman, 1995). However for the multi-robot systems, people have only recently started to investigate how to use this mechanism to coordinate teams of robots, and existing researches on this topic are of a bit limited.

Contract Net Protocol introduced by Smith (Smith, 1980) was the first economic model used to control multi-agent systems. Till now, major of the existing free market based multi-robot coordination methods are based upon this mechanism (Brian and Mataric, 2002) (Liu et al., 2004).

Of the previous free market based multi-robot coordination works, auction mechanism was the 
main idea (Brian and Mataric, 2002) (Liu et al., 2004) (Zlot et al., 2002). This method can coordinate multi-robot system achieving certain tasks effectively. But suppose of a multi-robot system, there is a complex atom task (we mean that the task can not be decomposed explicitly) which no single robot can accomplish separately, neither the above single item auction nor the combinatorial auctions mechanism can coordinate several robots to cooperate to execute. But if the bidding robots can perform some kind of negotiation and form a temporary group to submit bidding, the task may be performed by the cooperation of the group of robots.

We propose a set of formal descriptions to the multi-robot task allocation problem mainly focusing on the capability category aspect. Based upon it, a novel combinatorial bids multi-robot task allocation mechanism which enables several concerned robots to cooperate explicitly bidding for a relatively complex task is presented.

The rest of this paper is organized as follows. In the next section we give a formal description of robot and task capability category. Capability vector is defined in this section for both robot and task. In section III we describe the task accomplishment condition. Precedence order of tasks is discussed and a formal description is given. In section IV an auction and combinatorial bids method followed with some discussions is proposed. We conclude in section $\mathrm{V}$ and give a brief direction of future work.

\section{ROBOT AND TASK CAPABILITY CATEGORY}

Multi-robot coordination is a kind of comprehensive problem which contains many aspects to be studied. For the multi-robot task allocation aspect, there also need to be a general description of such kinds of problems so that we have a convenient tool to analyze and process them.

Generating a formal description to the multirobot task allocation problem becomes a very important basic work to the study of general aspects of this problem. In the multi-agent domain, there are many researchers studying this problem, but in the multi-robot aspect, few work is done. Gerkey (Brian and Mataric, 2003) gives a formal description and discusses ST/MT, SR/MR, IA/TA problem. Liu Lin (Liu et al., 2004) gives a formal description on the multi-robot task allocation problem, but this description is constraint to a kind of simple situation.

As we are concerned with the relatively complex task accomplishment, formal descriptions of robots and tasks should be discussed in detail.
This paper is based upon the previous method, but expands them focusing on capability category.

\subsection{Capability Category}

This paper focuses on the capability category of both robots and tasks. Robots may have different kinds of capabilities (e.g. sense capabilities, actuate capabilities, process capabilities, communication capabilities). And for a certain task, it may require a combination of capabilities to be accomplished. So capability serves as a very important role in the multi-robot task allocation problem.

Suppose there are together $m$ atom capabilities and each can be denoted as $c_{j}$. (By atom capability, we mean that it can not be divided into several sub capabilities.) Then the group of atom capabilities can be denoted as a set:

$$
C=\left\{c_{j}\right\}, \quad 1 \leq j \leq m
$$

\subsection{Capability Description of Robots}

In the earlier multi-robot research period, researchers focused on the loosely-coupled tasks such as homogeneous foraging (Mataric, 1993). But recently much attention on team coordination is focused on the complex and highly dynamics tasks employing heterogenous robots such as robot soccer (Asada et al., 1999).

Here suppose there are $\mathrm{n}$ heterogenous robots:

$$
r_{i}, \quad 1 \leq i \leq n
$$

For some robot $r_{i},(1 \leq i \leq n)$, its capability vector $C_{i}^{r}$ is defined as:

$$
\begin{aligned}
C_{i}^{r} & =\left[\begin{array}{cccc}
\alpha_{i 1} & 0 & \ldots & 0 \\
0 & \alpha_{i 2} & \ldots & 0 \\
\vdots & \vdots & \ddots & \vdots \\
0 & 0 & \ldots & \alpha_{i m}
\end{array}\right]\left[\begin{array}{c}
c_{1} \\
c_{2} \\
\vdots \\
c_{m}
\end{array}\right] \\
& \equiv A_{i} \cdot C
\end{aligned}
$$

Here $\alpha_{i j}$ corresponds to capability $c_{j}$ of robot $r_{i}$, $\alpha_{i j} \geq 0$. If robot $r_{i}$ does not have capability $c_{j}$, then $\alpha_{i j}$ equals 0 .

\subsection{Capability Description of Tasks}

As multi-robot task allocation has recently risen to prominence and become a key research in its own right, we should pay more attention to the task description problem.

For a whole mission, tasks can be regarded as a set of subgoals to be achieved. Tasks can be 
divided into independent tasks and interdependent tasks (Shehory and Kraus, 1998). This paper mainly deals with independent tasks which can be achieved independently of others. In case of where there are interdependencies among tasks, one solution is to combine dependent task into unified tasks, and then they can be regarded as independent tasks.

Suppose there are $l$ independent tasks:

$$
t_{k}, \quad 1 \leq k \leq l
$$

Then for task $t_{k},(1 \leq k \leq l)$, we define its corresponding capability vector $C_{k}^{t}$ as:

$$
\begin{aligned}
C_{k}^{t} & =\left[\begin{array}{cccc}
\beta_{k 1} & 0 & \ldots & 0 \\
0 & \beta_{k 2} & \ldots & 0 \\
\vdots & \vdots & \ddots & \vdots \\
0 & 0 & \ldots & \beta_{k m}
\end{array}\right]\left[\begin{array}{c}
c_{1} \\
c_{2} \\
\vdots \\
c_{m}
\end{array}\right] \\
& \equiv B_{k} \cdot C
\end{aligned}
$$

Here $\beta_{k j}$ corresponds to the needed capability $c_{j}$ of task $t_{k}, \beta_{k j} \geq 0$. If task $t_{k}$ need not capability $c_{j}$, then $\beta_{k j}$ equals 0 .

\section{TASKS ACCOMPLISHMENT CONDITION}

\subsection{Precedence Order of Tasks}

The separate tasks may have some kind of precedence order relationship which means that a specific task $t_{q}$ cannot be performed until another specific task $t_{p}$ has already been satisfied.

We denote the precedence order as:

$$
t_{p} \prec t_{q}, \quad 1 \leq p, q \leq l
$$

It means that $t_{p}$ is the predecessor of $t_{q}$, and $t_{q}$ is the successor of $t_{p}$.

All the tasks can be divided into several groups such that tasks in each group have the relationship of precedence order. The formal description is as follows:

$$
\begin{gathered}
t_{1}^{1} \prec t_{2}^{1} \prec \ldots \prec t_{l_{1}}^{1} \\
t_{1}^{2} \prec t_{2}^{2} \prec \ldots \prec t_{l_{2}}^{2} \\
\ldots \ldots \\
t_{1}^{m} \prec t_{2}^{m} \prec \ldots \prec t_{l_{m}}^{m} \\
\left\{t_{k}: 1 \leq k \leq l\right\}=\bigcup_{1 \leq q \leq m, 1 \leq p \leq l_{q}} t_{p}^{q}
\end{gathered}
$$

Consider a mission demonstrated in Fig.1, where the initial state is that seven blocks are scattered on the floor, the mission is to place these blocks

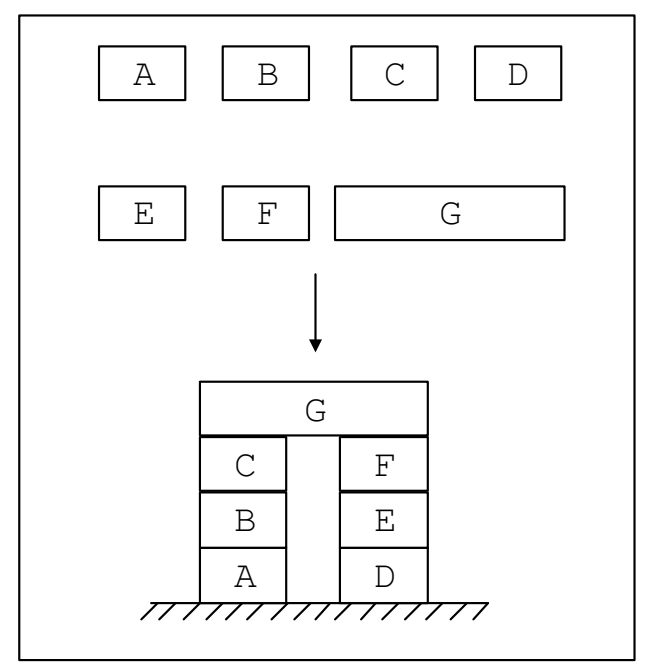

Fig. 1. A mission of blocks placement. In the upper of the figure is the initial state of the blocks: they are scattered on the floor. In the bottom is the final desired state.

in a certain order so that they achieve the final desired state displayed in the bottom.

We denote the placement action of block $\mathrm{A}$ as $t_{A}$, $\mathrm{B}$ as $t_{B}$, and so on. Then the precedence order relationships among this mission are as follows:

$$
\begin{gathered}
t_{A} \prec t_{B} \prec t_{C}, \\
t_{D} \prec t_{E} \prec t_{F}, \\
t_{C} \prec t_{G}, \quad t_{F} \prec t_{G}
\end{gathered}
$$

Block $\mathrm{G}$ can not be placed until block $\mathrm{C}$ and block $\mathrm{F}$ have been placed. And the placement of blocks $\mathrm{C}$ and $\mathrm{F}$ also depends on the other blocks of $\mathrm{A}, \mathrm{B}$, $\mathrm{C}, \mathrm{D}$. But blocks $\mathrm{C}$ and $\mathrm{F}$ have not any precedence order relationship which means that they can be placed in any order.

\subsection{Task Accomplishment Condition}

For some robot $r_{i}$, if it can accomplish some task $t_{k}$ alone, which means that it has all the capabilities the task needed, then there are:

$$
\alpha_{i j} \geq \beta_{k j}, \quad 1 \leq i \leq n, 1 \leq k \leq l, 1 \leq j \leq m(3)
$$

We denote the above conditions as:

$$
C_{i}^{r} \geq C_{k}^{t}
$$

For some robot $r_{i}$ and some task $t_{k}$, if there exists a certain capability $c_{j}$, such that:

$$
\alpha_{i j}<\beta_{k j}
$$


Then we claim that robot $r_{i}$ can not accomplish task $t_{k}$ alone. The above condition means that there is at least one kind of task capability which the robot does not have.

We also denote the above condition as:

$$
C_{i}^{r}<C_{k}^{t}
$$

\subsection{Cooperate Task Accomplishment}

One advantage of using multi-robot systems to accomplish tasks is that several robots can cooperate to achieve a complex task together. Using the capability category description provided in this paper, by cooperation, capabilities of the multirobot systems are more than any single one among them.

In some situations, none of the robots can accomplish a certain task $t_{k}$ separately, but several of them can cooperate to do so. That means inequation 6 holds for each robot, but the group capabilities are more than those of the task. If we define the group capability vector as $C_{N_{h}}$ (where $N_{h}$ will be described in 8), then the following inequation holds:

$$
C_{N_{h}} \geq C_{k}^{t}
$$

Task allocation to groups of robots is necessary when tasks cannot be performed by a single robot. It may also be beneficial when groups perform more efficiently than the single robot's performance.

Since complex task allocation among robots may be approached as a problem of assigning groups of robots to tasks, the partition of the robots into subgroups becomes the main issue. Therefore, the task allocation problem becomes similar to the set covering (SCP) and set partitioning (SPP) problems (Shehory and Kraus, 1998):

- Set Covering Problem

Suppose all the robots form a set $R$ :

$$
R=\left\{r_{i}, 1 \leq i \leq n\right\}
$$

Subsets of $R$ form a set $S$ :

$$
S=\left\{N_{1}, \ldots, N_{p}\right\},
$$

such that $N_{h} \subseteq R$ and $S \subseteq 2^{R}, 1 \leq h \leq p$

A set-cover is defined as any set $S^{\prime}$ :

$$
S^{\prime} \subseteq S \text {, such that } \bigcup_{N_{h} \in S^{\prime}} N_{h}=R
$$

The members of $S^{\prime}$ are the covering sets.
- Set Partition Problem

If set $S^{\prime}$ is a set covering of $R$, and the members of $S^{\prime}$ are also pairwise disjoint, that means:

$$
\begin{gathered}
N_{i} \cap N_{j}=\emptyset \\
\forall N_{i}, N_{j} \in S^{\prime}, i \neq j, 1 \leq i, j \leq p
\end{gathered}
$$

then $S^{\prime}$ is a set-partitioning of R.

If set $S^{\prime}$ is a set-covering but not a setpartitioning of $\mathrm{R}$, we can claim that:

$$
\begin{gathered}
\exists r_{k} \in R, \exists N_{i}, N_{j} \in S^{\prime}, \\
1 \leq k \leq n, i \neq j, 1 \leq i, j \leq p \\
\text { so that } r_{k} \in N_{i} \cap N_{j}
\end{gathered}
$$

In the multi-robot task allocation domain, this means that robot $r_{k}$ may perform more than one tasks at the same time and in some situations this assumption may hold (e.g. a single robot may surveille more than one objects at the same time).

Gerkey (Brian and Mataric, 2003) gives a taxonomy of multi-robot task allocation problems. According his taxonomy, the above situations are corresponding to multi-task robots (MT) and single-task robots (ST).

When two or more robots cooperate to perform a certain task, their whole capabilities depend on their capability attributes. We can divide capabilities into two types:

- Addition of Capability If some capability of a subset of robots is the sum of the capabilities of all the robots in the subset, then we claim that this capability is additivity. We assume in subset $N_{h}$ capability $c_{j}$ is additivity, then:

$$
\alpha_{N_{h} j}=\sum_{r_{i} \in N_{h}} \alpha_{i j}
$$

For instance, in the multi-robot cooperate box pushing environment, strength is an additivity capability. When two robots cooperate to push a box in the same direction, we can simply add their strength as their whole one.

- Union of Capability

However some capabilities can not simply be added together to obtain the whole subset's capability. For instance, vision capabilities of different robots may be not simply the sum of the separate robots, but the union of them:

$$
\alpha_{N_{h} j}=\bigcup_{r_{i} \in N_{h}} \alpha_{i j}
$$

If a subset of robots $N_{h}$ can accomplish some task $t_{k}$ by cooperation, then there are:

$\alpha_{N_{h} j} \geq \beta_{k j}, \quad 1 \leq h \leq p, 1 \leq k \leq l, 1 \leq j \leq m(9)$ 
We name the above conditions cooperate task accomplishment conditions and denote them as:

$$
C_{N_{h}}^{r} \geq C_{k}^{t}
$$

\section{AUCTION AND COMBINATORIAL BIDS METHOD}

Free market based mechanism is proven an efficient method for both multi-agent and multirobot systems coordination.

\subsection{Idea of Combinatorial Bids Method}

Cost and reward function definitions (Liu et al., 2004) are important concepts when implementing the free market method. We expand them to the case of robot subsets.

- Cost function

This function is a mapping from a resource set to a positive real number.

$$
\operatorname{Cost}_{N_{h}, k}
$$

where $N_{h} \subseteq R$ and $t_{k} \in T$.

- Reward function

This function is a mapping from the task set $T$ to a positive real number.

$$
R e w_{N_{h}, k}
$$

where $N_{h} \subseteq R$ and $t_{k} \in T$.

- Benefit function

When a subset of robots or even only one robot performs a certain task, it will gain a benefit $B$.

$$
B_{N_{h}, k}
$$

Of the previous free market based multi-robot coordination works, auction mechanism is the main idea (Brian and Mataric, 2002) (Liu et al., 2004) (Zlot et al., 2002).

The typical auction multi-robot task allocation method can be divided into three main steps. In the first step, task auctioneers announces the task to the surroundings. Then the second step, each candidate robot bids for its expected task. At last, after sufficient time has passed, the auctioneers process the bids, determine the winners and allocate the corresponding tasks to them.

Some researchers bring forward the so called combinatorial auction method in the multi-agent domain (Tuomas, 2002) (Sven and Rakesh, 2003). The idea of combinatorial auction is that some candidate robot may gain more benefit by bidding on sets or bundles of items (Sven and Rakesh,
2003). Until now, this mechanism is only implemented in the multi-agent domain (an exception is (Berhault et al., 2003)). Here we take into account of the antithesis of combinatorial auction: combinatorial bids (CB).

This mechanism mainly includes six steps: task announcement, bids submission, task pre award, bids combination, com-bids and task allocation.

The merit of such a method is that even for each robot $r_{i}$ in $R$ inequation 6 holds, but if there is some subset $N_{h}$ that inequation 10 holds, then they can still accomplish task $t_{k}$ by cooperation.

\subsection{Discussion of the Method}

Initial validation of the proposed combinatorial bids based multi-robot task allocation mechanism was based upon an improved simulation environment of MuRos (Luiz and Mario, 2001) (Luiz et al., 2002). As this tool is source code open, based upon it we add two new classes of "CRobotAuction" and "CBoxAuction" representing the robots and tasks for simulation.

The simulation scenario is a complex consume task a bit like that of (Balch and Arkin, 1994). Fig.2 demonstrates a snapshot of the simulation scenario where many boxes and robots of different capability vectors are scattered randomly. The task is for the robots to cooperatively consume all of the boxes.

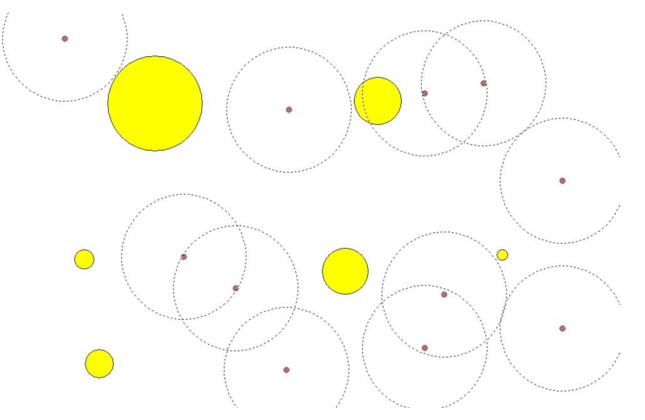

Fig. 2. A snapshot of the simulation scenario.

We define several kinds of consume tasks in the scenario. Each kind of them is corresponding to a combination of capabilities. The robots here are also heterogenous in that they have different capabilities. All the tasks and robots capabilities are defined in a description file which can be modified as experiment needed.

Several aspects of the proposed combinatorial bids coordination mechanism are studied. With the typical auction method, once the robots accomplish the tasks that can be achieved by a single robot, they do not attempt to explicitly cooperate to take on the left tasks. So they become idle. But 
with the combinatorial bids method we proposed, once the single-robot tasks are accomplished, the robots are still active seeking for chances to explicitly cooperate to accomplish the rest tasks.

\section{CONCLUSION AND FUTURE WORK}

We have presented a formal description to the multi-robot task allocation problem mainly focusing on the capability category aspect. Both robot and task capability vectors are defined. Based upon the these, both single and multi-robot cooperative task accomplishment conditions are discussed.

As the typical auction (or combinatorial auctions) based mechanisms have some inherent disadvantages, we propose a novel combinatorial bids method based upon the capability category and task accomplishment condition.

As the capability category description method proposed is just a initial work to our research, we will study more different aspects of the multirobot task allocation problem based upon this formal description in the future work. Another direction is to implement the coordination methods into our real robot team.

\section{REFERENCES}

Arai, Tamio, Pagello Enrico and Lynne E Parker (2002). Advances in multirobot systems. IEEE Transacitons on Robotics and Automation 18(5), 655-661.

Asada, Minoru, Hiroaki Kitano, Itsuki Noda and Manuela Veloso (1999). Robocup: today and tomorrow - what we have learned. Artificial Intelligence 110(2), 193-214.

Balch, Tucker and Ronald C. Arkin (1994). Communication in reactive multiagent robotic systems. Autonomous Robots 1(1), 27-52.

Berhault, M, H Huang, P Keskinocak, S Koenig, W Elmaghraby, P Griffin and A Kleywegt (2003). Robot exploration with combinatorial auctions. In: Proceedings of the International Conference on Intelligent Robots and Systems.

Brian, P Gerkey and Maja J Mataric (2002). Sold!: Auction methods for multirobot coordination. IEEE Transacitons on Robotics and Automation 18(5), 758-768.

Brian, P Gerkey and Maja J Mataric (2003). A formal analysis and taxonomy of task allocation in multi-robot systems. Technical Report CRES-03-013. University of Southern California, USA.

Brian, P Gerkey and Maja J Mataric (2004). On role allocation in robocup. In: RoboCup2003: Robot Soccer World Cup VII (Daniel Polani,
Brett Browning, Andrea Bonarini and Kazuo Yoshida, Eds.). Vol. LNAI3020. SpringerVerlag. pp. 43-53.

Cao, Y Uny, S Fukunaga Alex and B Kahng Andrew (1997). Cooperative mobile robotics: antecedents and directions. Autonomous Robots 4(1), 7-27.

Liu, Lin, Lei Wang, Zhi Qiang Zheng and Zeng Qi Sun (2004). A learning market based layered multi-robot architecture. In: Proceedings of the 2004 IEEE International Conference on Robotics and Automation. IEEE. New Orleans, LA, USA. pp. 3417-3422.

Luiz, Chaimowicz and Campos Mario (2001). Simulating loosely and tightly coupled multirobot cooperation. In: Proceedings of the fifth Brazilian Symposium on Intelligent Automation. Canela, RS, Brazil.

Luiz, Chaimowicz, F M Campos Mario and Vijay Kumar (2002). Dynamic role assignment for cooperative robots. In: Proceedings of the 2002 IEEE International Conference on Robotics and Automation. IEEE. Washington, DC, USA. pp. 293-298.

Mataric, Maja J. (1993). Designing emergent behaviors: from local interactions to collective intelligence. In: Proceedings of the second international conference on From animals to animats 2: simulation of adaptive behavior. MIT Press. pp. 432-441.

Parker, Lynne E (1998). Alliance: An architecture for fault tolerant multirobot cooperation. IEEE Transactions on Robotics and Automation 14(2), 220-240.

Shehory, Onn and Sarit Kraus (1998). Methods for task allocation via agent coalition formation. Artificial Intelligence 101(1-2), 165200.

Smith, P. G. (1980). The contract net protocol: High level communication and control in a distributed problem solver. IEEE Transactions on Computers C-29(12), 1104-1113.

Sven, Vries and V. Vohra Rakesh (2003). Combinatorial auctions: A survey. INFORMS Journal on Computing 15(3), 284-309.

Tuomas, Sandholm (2002). Algorithm for optimal winner determination in combinatorial auctions. Artificial Intelligence 135(1-2), 1-54.

Wellman, M (1995). The economic approach to artificial intelligence. ACM Computing Survey $7(3), 758-768$.

Zlot, Robert, Anthony Tony Stentz, M.Bernardine Dias and Scott Thayer (2002). Multi-robot exploration controlled by a market economy. In: Proceedings of the 2002 IEEE International Conference on Robotics and Automation. IEEE. Washington, DC, United States. pp. 3016-3023. 